\title{
Evaluation of Maximum Voltage or Maximum Link Distance on Implantable Devices
}

\author{
Paulo Cesar Crepaldi, Tales Cleber Pimenta, \\ Robson Luiz Moreno and Rômulo Mota Volpato
}

Additional information is available at the end of the chapter

http://dx.doi.org/10.5772/48648

\section{Introduction}

The current technology allows the implementation of electronic devices under the skin for therapeutic purposes. As a result the wireless technology can be used to monitor some illness and mitigate the patient's discomfort. As an example, consider the case of diabetes patients that must undergo to daily painful blood collections to monitor the blood sugar levels. Therefore an electronic device capable of measuring the blood sugar level and transmit the result to an external device would greatly improve the quality of life of those patients. Nevertheless, the placement of an implantable device requires a minor surgery.

Once the implantable device is placed in a subject, the battery lifetime and its charge will dictate the system lifespan [1].

Consequently, it could be used the passive target technology, in which the device contains its own energy source. Thus the limitation imposed by batteries would not exist in addition to size reduction to the device. The radio frequency identification - RFID technology fits perfectly into that application.

An important parameter to be analyzed is the operation frequency. The communication frequency between the external reader and the implantable device should suffer the smallest interference due to the human tissue. The frequency of $13.56 \mathrm{MHz}$ offers low attenuation and can be chosen for implantable devices. This chapter discusses the energy transfer from reader to tag by inductive coupling and its limitations. It presents a way to evaluate the target voltage provided by the inductive coupling by using basic circuit theory. 


\section{Basic inductive coupling circuit}

Figure 1 shows a typical inductive coupling system. Observe that the system is composed by the reader and the tag. The reader is used to receive data from the tag and also to transfer energy to it.

It is chosen series resonance configuration, as shown in Figure 2, in order to raise the voltage at inductor $\mathrm{L}_{1}$, since voltage in a series resonance inductor is larger than the voltage provided by the source. From basic circuit theory and by observing Figure 3, the impedance seen by the reader is given as:

$$
Z=R_{1}+\left(j \omega L_{1}-j \frac{1}{\omega C_{1}}\right)
$$

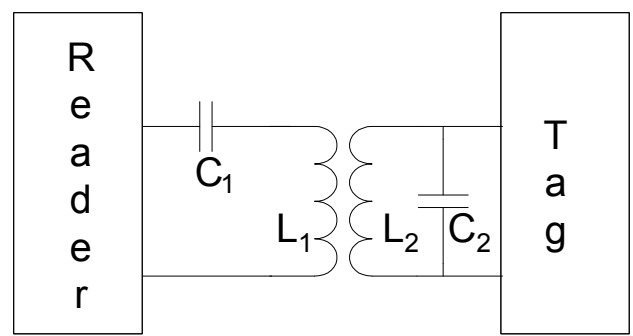

Figure 1. Typical inductive coupling system.

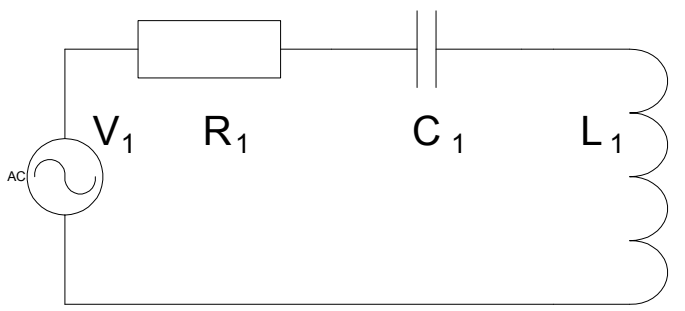

Figure 2. Simplified inductive coupling circuit at the reader.

The resonance, for low frequency operation, is given as:

$$
\omega L_{1}=\frac{1}{\omega C_{1}}
$$

However impedance $\mathrm{Z}$ becomes resistive and equal to $R_{1}$ that represents the inductor series resistance. Therefore, the reader impedance depends on resistance $R_{1}$ and voltage the inductor becomes:

$$
E_{1}=j I \omega L_{1}=j \frac{V_{1} \varpi L_{1}}{R_{1}}=j Q V_{1}
$$


At this point it is important to define the quality factor of the resonant circuit, $Q=w L_{1} / R_{1}$. It defines the ratio of stored and dissipated energy in an inductor. It demonstrates that the reader efficiency increases by using series resonance, as summarized by expression (4).

$$
\left|E_{1}\right|=\left|V_{1}\right| Q
$$

The reader receives data and power by the inductive coupling through $L_{1}$ and $L_{2}$. The reader takes advantage of the parallel resonance and high impedance that occurs at frequencies near the resonance. It indicates that, at resonance, the voltage over the $L C$ circuit is high and can be used to power up the target. Therefore the inductive coupling allows the use of passive target concept, or self-powering. Nevertheless tuned circuits have multiple resonances that cause a reduction in the energy transfer from reader to target. Thus a better understanding of the influence of resonance circuits may help to determine precisely the supply voltage at the target.

\section{Concept of inductance}

A current flowing in a straight infinite conductor generates a magnetic flow density (or magnetic induction) proportional to the current. That relationship was observed by Biot Savart, as given by (5).

$$
\vec{B}=\frac{\mu_{0}}{4 \pi} \int i \frac{d \vec{l} X \vec{a}_{r}}{|\vec{r}|^{2}}
$$

where:

$d \vec{l}$ represents an elemental piece of conductor,

$\vec{a}_{r}$ represents the unit directional vector,

$|\vec{r}|^{2}$ represents the module of distance where the magnetic flow intensity is calculated,

$\mu_{0}$ is the vacuum permeability constant.

From (5), there will be a magnetic flow area around the conductor. The magnetic flow is given by (6)

$$
\phi=\int \vec{B} d \vec{S}
$$

where:

$\vec{B}$ - represents the magnetic induction vector,

$d \vec{S}$ - represents the area vector inside the magnetic induction vector.

In that line, the magnetic flow is proportional to the current flowing in the conductor and there is a linear relationship between them, known as inductance, as shown by (7). 


$$
L \frac{d i}{d t}=\frac{d \phi}{d t} \Rightarrow L=\frac{d \phi}{d i}
$$

The premise of infinite and straight conductor of the Biot Savart law is not obeyed in the construction of inductors, thus the inductance parameter of a component will depend on many factors such as geometric shape, number of coils and resistance of the conductor.

\section{Concept of mutual inductance}

Whenever two inductors are close, the magnetic flow generated by one influences the flow in the other, as indicated in Figure 3.

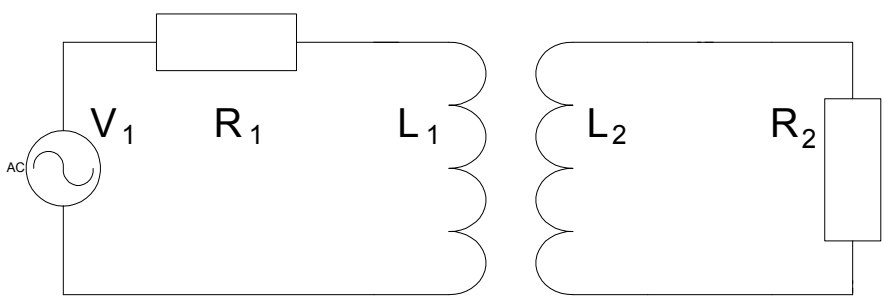

Figure 3. Simplified inductive coupling.

Therefore, a time varying current i1 flowing on inductor 1 generates a magnetic flow $\phi 21$ into inductor 2. The density of magnetic flow that reaches inductor 2 depends on the position and distance between the inductors. Thus mutual inductance is defined as the number of coils of inductor 2 that receives the magnetic flow generated by inductor 1 , divided by current i1, as shown by (8).

$$
M_{21}=\frac{N_{2} \phi_{21}}{i_{1}}
$$

where :

1. $N_{2}$ is the number of coils of inductor 2 ,

2. $i_{1}$ is the current on inductor 1 ,

3. $\phi_{12}$ is the magnetic flow on inductor 2 due to inductor 1 ,

4. $\quad M$ is mutual inductance between inductors 1 and 2 .

If there is any current on inductor 2, the same phenomenon occurs, as given by (9).

$$
M_{12}=\frac{N_{1} \phi_{12}}{i_{2}}
$$

Consider two inductors of length $D$ and sectional areas $A_{1}$ and $A_{2}$, much smaller than $D_{\text {, }}$ number of coils $N_{1}$ and $N_{2}$ for inductors 1 and 2, respectively, and placed on the same vertical axis, as shown in Figure 4 . As can be observed, inductor 1 is placed inside inductor 2 
and they have the same orientation. If it is applied a current into inductor 2, the mutual inductance is given by (9).

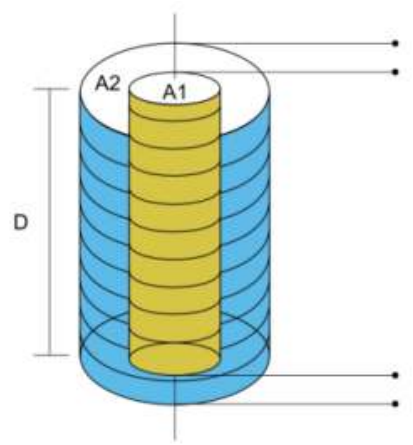

Figure 4. Long inductors coupled on the same axis.

If the magnetic field is uniform inside inductor 2, then flow $\phi_{12}$ can be taken as (10).

$$
\phi_{12}=B_{2} A_{1}
$$

Expression (10) can be placed into (9), as:

$$
M_{12}=\frac{N_{1} A_{1} B_{2}}{i_{2}}
$$

By considering that the inductors core is air and the coils are composed of close and simple layers, the Biot e Savart expression can be used, where:

$$
B_{2}=\frac{\mu_{0} N_{2} i_{2}}{d}
$$

Thus, by combining (11) and (12):

$$
M_{12}=\frac{N_{1} N_{2} \mu_{0} A_{1}}{d}
$$

From the analysis of (13) it can be observed that for the auto inductance, the number of coils is the same, thus the expression becomes:

$$
L=\frac{\mu_{0} N^{2} A}{d}
$$

For the inductor, the coupling will be taken in its own area, meaning that they are equal, thus:

$$
L_{1}=\frac{\mu_{0} N_{1}^{2} A}{d}
$$




$$
L_{2}=\frac{\mu_{0} N_{2}^{2} A}{d}
$$

By combining (15) and (16), one obtains:

$$
M=\frac{\mu_{0} A}{d} \sqrt{\frac{L_{1} d}{A \mu_{0}}} \sqrt{\frac{L_{2} d}{A \mu_{0}}}=\sqrt{L_{1} L_{2}}
$$

In real case, areas $A_{1}$ and $A_{2}$ are not equal, indicating that the magnetic flow is not covered completely by both inductors. Therefore, the coupling factor is reduced, as given by:

$$
M=k \sqrt{L_{1} L_{2}}
$$

where $k$ is the magnetic coupling factor.

\section{Inductive coupling with simple resonance}

Although we have discussed inductive coupling between reader and target, at resonance, that characterize a double resonance, we can use simple resonance. In this configuration the reader presents just an inductor magnetically coupled to the inductor at the tag. The simplified circuit is shown in Figure 5. Observe the resonant circuit is positioned at the tag. In order to simplify the analysis, consider the circuit in free space. By considering also inductors without loss, the circuit of Figure 5 can be equated by using (2), resulting into (19) and (20).

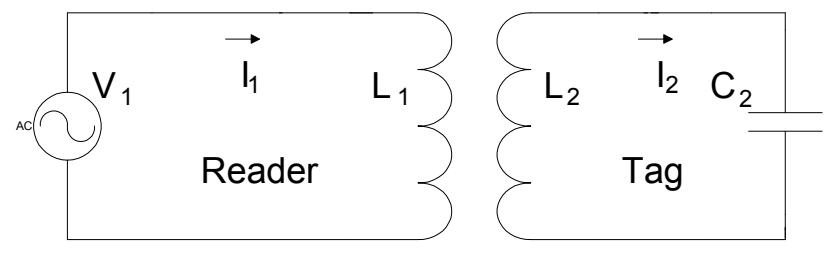

Figure 5. Inductive coupling with simple resonance at the tag.

$$
\begin{gathered}
V_{1}=I_{1} j \varpi L_{1}-I_{2} j \varpi M \\
0=I_{2}\left(j \varpi L_{2}+\frac{1}{j w C_{2}}\right)-I_{1} j \varpi M
\end{gathered}
$$

where $M$ is the mutual inductance between inductors $L_{1}$ and $L_{2}$.

From (20):

$$
I_{2}=\frac{I_{1} j \varpi M}{j \varpi L_{2}+\frac{1}{j \varpi C_{2}}}
$$


By replacing (18) into (21):

$$
I_{2}=\frac{I_{1} j \varpi k \sqrt{L_{1} L_{2}}}{j \varpi L_{2}+\frac{1}{j \varpi C_{2}}}
$$

Now, replacing (22) into (19):

$$
V_{1}=I_{1} j \varpi L_{1}-\frac{I_{1} j \varpi k \sqrt{L_{1} L_{2}} j \varpi k \sqrt{L_{1} L_{2}}}{j \varpi L_{2}+\frac{1}{j \varpi C_{2}}}
$$

Thus, the impedance seen by the generator is:

$$
Z_{1}=\frac{V_{1}}{I_{1}}=j \varpi L_{1}+\frac{\varpi^{2} k^{2} L_{1} L_{2}}{j \varpi L_{2}+\frac{1}{j \varpi C_{2}}}
$$

From the analysis of (24), it can be observed that the inductance seen by the reader is inductance $L_{1}$ added to impedance $Z_{a}$ reflected from the target, and represented by:

$$
Z_{a}=\frac{\varpi^{2} k^{2} L_{1} L_{2}}{j \varpi L_{2}+\frac{1}{j \varpi C_{2}}}
$$

Therefore, by using the same analysis, the impedance $Z_{b}$ reflected on inductor $L_{2}$ is:

$$
Z_{b}=\frac{\varpi^{2} k^{2} L_{1} L_{2}}{j \varpi L_{1}}
$$

The equivalent circuit reflected at the target is shown in Figure 6.

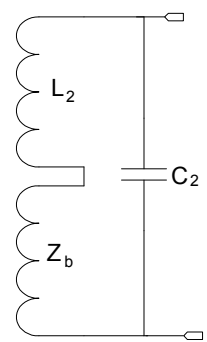

Figure 6. Equivalent circuit reflected at the inductor $L 2$.

By considering the equivalent circuit at resonance, then: 


$$
0=\left(j \varpi L_{2}+\frac{\varpi^{2} k^{2} L_{1} L_{2}}{j \varpi L_{1}}+\frac{1}{j \varpi C_{2}}\right) I_{2}
$$

From the development of (27):

$$
0=j \varpi L_{2}\left(1-k^{2}\right)+\frac{1}{j \varpi C_{2}}
$$

From (28), the resonance frequency is given as:

$$
F o_{1}=\frac{1}{2 \pi \sqrt{L_{2}\left(1-k^{2}\right) C_{2}}}
$$

Thus the resonance frequency at the target was modified by the term $r$ given by:

$$
r=\frac{1}{\sqrt{1-k^{2}}}
$$

Consequently, the resonant frequency varies according to the coupling factor $k$. Thus, the distance between the inductors and their relative orientation can modify the amount of energy transferred by the inductive coupling. In order to validate equation (29), it was run a Spice simulation with a coupling factor ranging from 0.1 to 0.3 , as shown in Figure 7 . It can be observed that resonance frequency increases along with the coupling factor. As an initial condition, $L_{1}$ and $L_{2}$ were taken as $3,5 \mu \mathrm{H}$ and capacitor $C_{1}$ equals to $38 \mathrm{pF}$. Observe, from Figure 7 , that voltage on inductor $L$ increases along with the coupling factor.

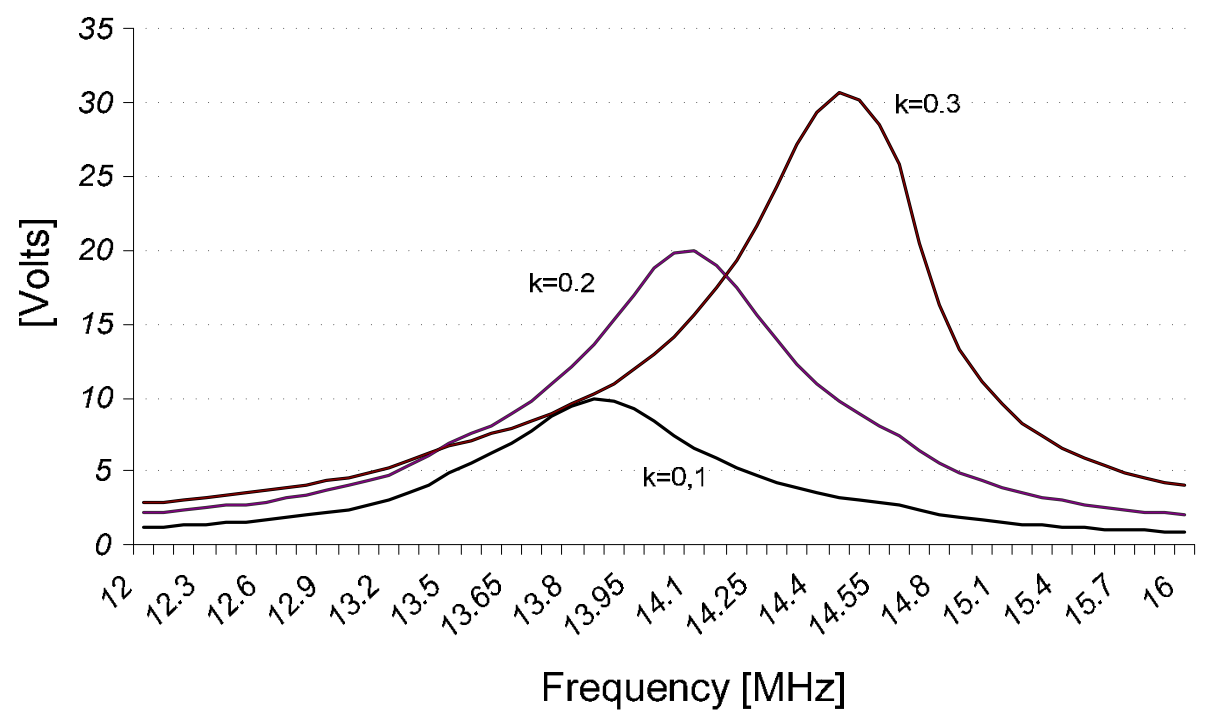

Figure 7. Spice simulation of coupling factor. 


\section{Inductive coupling with double resonance}

There are two tuned circuits in the double resonance, where the primary is the reader and the secondary is the tag. Figure 8 presents the basic configuration for the double resonance inductive coupling.
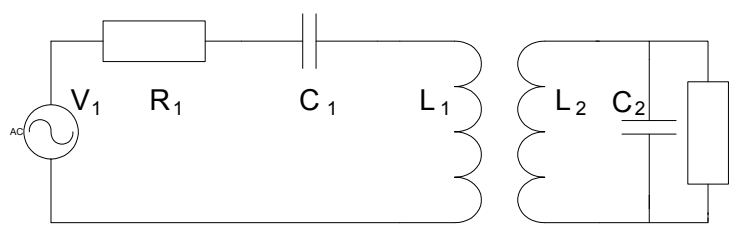

Figure 8. Double resonance inductive coupling.

From the analysis of Figure 8 and by considering free space and inductors without loss, then:

$$
\begin{aligned}
& V_{1}=I_{1}\left(j \varpi L_{1}+\frac{1}{j \varpi C_{1}}\right)-I_{2} j \varpi M \\
& 0=I_{2}\left(j \varpi L_{2}+\frac{1}{j \varpi C 2}\right)-I_{1} j \varpi M
\end{aligned}
$$

From (32) it can be obtained that:

$$
I_{2}=\frac{j \varpi M I_{1}}{j \varpi L_{2}+\frac{1}{j \varpi C_{2}}}
$$

By replacing (33) into (31) one obtains:

$$
\left.V_{1}=I_{1}\left(j \varpi L_{1}+\frac{1}{j \varpi C_{1}}\right)+\frac{\varpi^{2} M^{2}}{j \varpi L_{2}+\frac{1}{j \varpi C_{2}}}\right)
$$

Therefore, the impedance seen by the generator is:

$$
Z=\frac{V_{1}}{I_{1}}=\left(j \varpi L_{1}+\frac{1}{j \varpi C_{1}}\right)+\frac{\varpi^{2} M^{2}}{\left(j \varpi L_{2}+\frac{1}{j \varpi C_{2}}\right)}
$$

It can be rewritten as:

$$
Z=j\left\{\frac{\left(1-w^{2} L_{1} C_{1}\right)\left(1-w^{2} L_{2} C_{2}\right)+w^{4} M^{2} C_{1} C_{2}}{w C_{1}\left(1-w^{2} L_{2} C_{2}\right)}\right\}
$$


Expression (36) allows the behavior analysis of the impedance at the generator, as shown in Figure 8. As previously shown, the mutual inductance depends on the coupling factor. Therefore, it can be verified the influence of the coupling factor in the response of a double tuned circuit. It was run a Spice simulation, as shown in Figure 9, where the coupling factor ranged from 0.1 to 0.2 , and the frequency varied from 12 to $16 \mathrm{MHz}$. As an initial condition, $L_{1}$ and $L_{2}$ were taken as $3,5 \mu \mathrm{H}$ and capacitors $C_{1}$ and $C_{2}$ are equal to $38 \mathrm{pF}$. In that case, the resonance frequencies at primary and secondary are equal and it will be taken as an individual resonance.

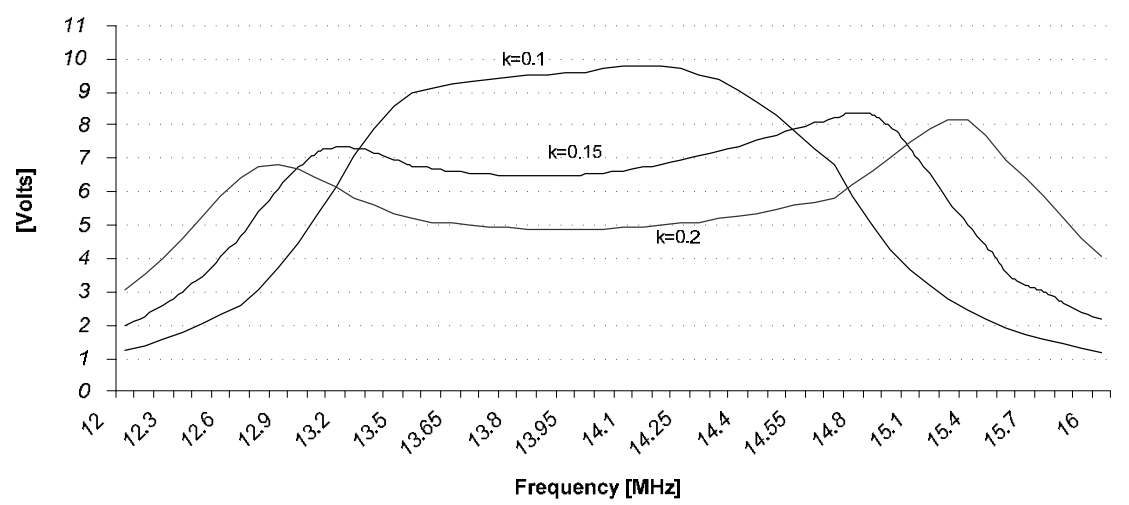

Figure 9. Spice simulation of double resonance.

From Figure 9 it can be observed that larger coupling factor causes larger deviation of the resonance frequency from the individual frequency. The energy transfer by RF coupling is directly dependent on the variation of the coupling and consequently dependent on the approaching between reader and tag, in other words orientation of the reader and tag inductances strongly changes the double resonance between reader and tag.

\section{Mutual inductance measurement method}

According to [3] and [4], the mutual inductance between two inductors can be measured. Considering two magnetically coupled inductors, as shown in Figure 10, the inductance seen by the generator is given as:

$$
L_{S}{ }^{\prime}=L_{1}+M_{12}+M_{21}
$$
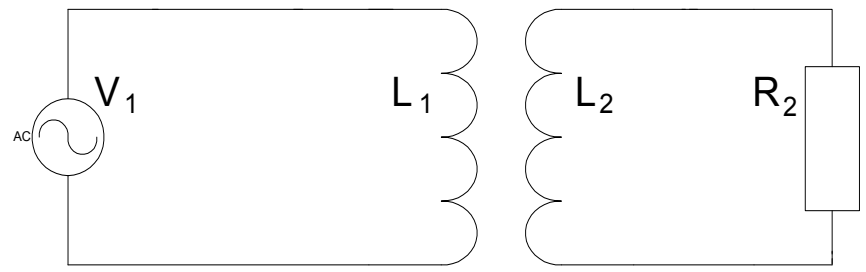

Figure 10. Magnetically coupled circuits. 
Thus, the inductance seen at the generator terminals is the inductance plus the mutual inductance of inductor 1 over inductor 2, plus the mutual inductance of inductor 2 over inductor 1. Now, considering the series association, as shown in Figure 11, the total inductance is given as:

$$
L_{s}{ }^{\prime}=L_{1}+L_{2}+M_{21}+M_{12}
$$

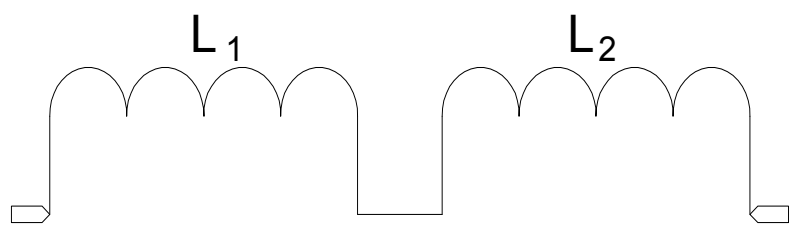

Figure 11. Series association of inductors.

If inductances $L_{1}$ and $L_{2}$ are associated in series, as shown in Figure 11, the resulting inductance is the sum of the individual inductance and the mutual inductances. If the measurement method is altered, as shown in Figure 12, where one inductor is inverted, the new series inductance is given as:

$$
L_{s}{ }^{\prime \prime}=L_{1}+L_{2}-M_{12}-M_{21}
$$

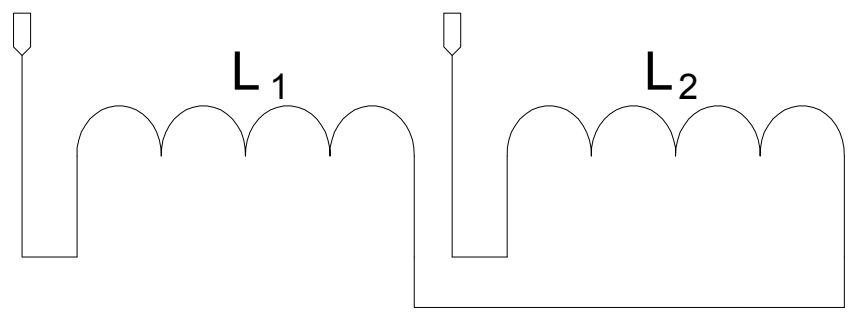

Figure 12. Series association of inductors, in which one inductor is inverted.

Now, subtracting $L^{\prime}$ from $L^{\prime \prime}$ results:

$$
L_{1}+L_{2}+M_{12}+M_{21}-L_{1}-L_{2}+M_{12}+M_{21}=L_{s}{ }^{\prime}-L_{s}{ }^{\prime}
$$

By considering $M_{12}=M_{21}=M$, then:

$$
M=\frac{L_{S}{ }^{\prime}-L_{S}{ }^{\prime}}{4}
$$

Once $L_{1}$ and $L_{2}$ are known, the coupling factor can be found as:

$$
k=\frac{M}{\sqrt{L_{1} L_{2}}}
$$




\section{Model for the energy transfer based on the classic circuit theory}

The energy transfer between reader and tag in free space can be modeled using the classic circuit theory. Consider Figure 13 that represents the magnetic coupling between reader and tag.

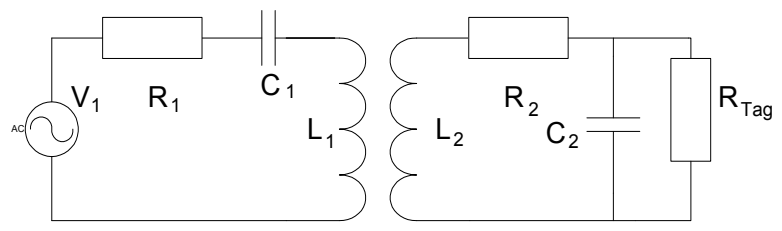

Figure 13. Magnetic coupling between reader and tag.

Observe that resistor $R_{2}$ represents the losses on $L_{2}, R_{\text {tag }}$ represents the load at the tag and $R_{1}$ represents the losses at inductor $L_{1}$. The analysis can be greatly simplified if the reader circuit can be converted into the series equivalent circuit, since the reader load could be represented as the resistance losses of capacitor $C_{2}$. Therefore, the quality factor of capacitor $C_{2}$ can be expressed in terms of its losses resistance, as indicated in Figure 14. The equivalence between series and parallel circuit is granted if the real and imaginary parts are equal, thus:

$$
\begin{gathered}
y_{p}=G_{p}+j \omega C_{p} \\
R_{s}=\frac{G_{p}}{G_{p}{ }^{2}+\left(\omega C_{p}\right)^{2}} \\
C_{s}=\frac{G_{p}{ }^{2}+\left(\omega C_{p}\right)^{2}}{\omega^{2} C_{p}} \\
G_{p}=\frac{1}{R_{p}}
\end{gathered}
$$

Figure 14. Capacitive equivalent circuits.

That approximation allows the conversion of tag load $R_{p}$ into $R_{s}$, as given by (44). The approximation is possible since the capacitor presents low losses at the frequency range. 
Thus, tag load provides the larger contribution in the energy dissipation in the magnetic coupling between reader and tag.

Observe also that the equivalent capacitor given by (45) is frequency dependent that implies in another source of variation in the resonance frequency at the tag. Based on that premise, the equivalent circuit is shown in Figure 15.
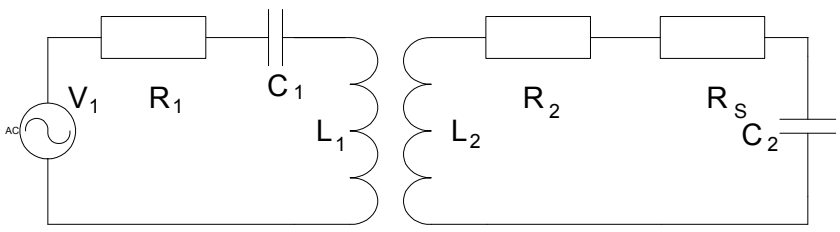

Figure 15. Modified equivalent circuit.

The circuit of Figure 15 can be equated as:

$$
R_{t}=R_{2}+R_{s}
$$

Thus:

$$
V_{1}=I_{1}\left(R_{s}+j \omega L_{1}+\frac{1}{j \omega C_{1}}\right)-I_{2} j \omega M
$$

where $M$ is the mutual inductance between $L_{1}$ and $L_{2}$.

$$
0=-I_{1} j \omega M+I_{2}\left(R_{t}+j \omega L_{2}+\frac{1}{j \omega C_{2}}\right)
$$

Combining (48) and (49) results into:

$$
V_{2}=\frac{V_{1} M}{\left[(\omega M)^{2}+\left(R t+j \omega L_{2}+\frac{1}{j \omega C_{2}}\right)\left(R_{s}+j \omega L_{1}+\frac{1}{j \omega C_{1}}\right)\right] C_{2}}
$$

It can be observed from (5) the possibility of predicting the voltage at the tag, under free space conditions. Nevertheless, in real application, the tag shall be placed inside a human being, usually in the belly area, in order to measure any biological data. Since pork tissue presents electromagnetic properties similar to human tissue, it will be used in the measurements.

\section{Comparison between simulated and measured data for free space}

It was used the test set up shown in Figure 16, where $L_{1}=1.77 \mathrm{uH}$ and $L_{2}=5.4 \mathrm{uH}$. Observe that the inductors are axially aligned. That orientation was chosen to achieve the best magnetic coupling, considering the ratio between length and diameter of the inductor. The 
inductors series resistances $R_{1}=1.14 \Omega$ and $R_{2}=2.2 \Omega$ (at $13.56 \mathrm{MHz}$ ) were obtained using a network analyzer. The experimental measurements and the simulations were conducted considering a generator having a $1 \mathrm{~V}$ and a $50 \Omega$ internal impedance. The tag load was taken as a $1 \mathrm{k} \Omega$ resistor. Table 1 presents the measurement results.

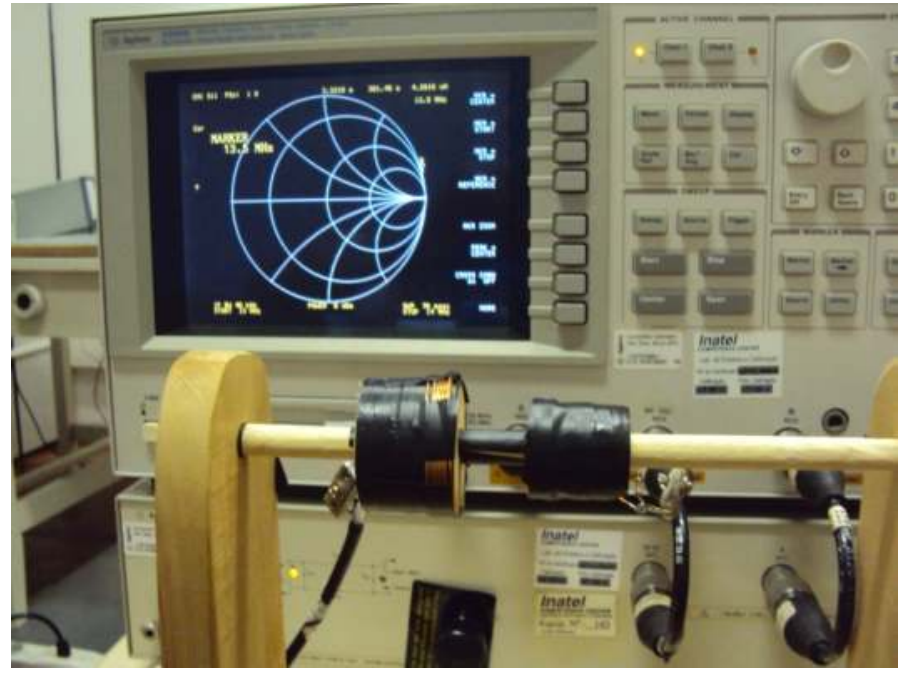

Figure 16. Free space measurement test set up.

\begin{tabular}{|c|c|c|}
\hline Measurement & Distance $L_{1}-L_{2}$ & $M$ \\
\hline $\begin{array}{l}L^{\prime}=10.00 \mathrm{uH} \\
L^{\prime \prime}=6.33 \mathrm{uH}\end{array}$ & $5 \mathrm{~mm}$ & $0.917 \mathrm{uH}$ \\
\hline $\begin{array}{l}L^{\prime}=9.48 \mathrm{uH} \\
L^{\prime \prime}=6.71 \mathrm{uH}\end{array}$ & $10 \mathrm{~mm}$ & $0.692 \mathrm{uH}$ \\
\hline $\begin{array}{c}L^{\prime}=9.13 \mathrm{uH} \\
L^{\prime \prime}=7.00 \mathrm{uH}\end{array}$ & $15 \mathrm{~mm}$ & $0.532 \mathrm{uH}$ \\
\hline $\begin{array}{c}L^{\prime}=8.74 \mathrm{uH} \\
L^{\prime \prime}=7.26 \mathrm{uH}\end{array}$ & $20 \mathrm{~mm}$ & $0.370 \mathrm{uH}$ \\
\hline $\begin{array}{c}L^{\prime}=8.55 \mathrm{uH} \\
L^{\prime \prime}=7.40 \mathrm{uH}\end{array}$ & $25 \mathrm{~mm}$ & $0.287 \mathrm{uH}$ \\
\hline
\end{tabular}

Table 1. Mutual inductance measurements.

It was conducted a set of simulations of equations (44), (45) and (50) for the range of 12 to 16 $\mathrm{MHz}$ and the result is shown in Figure 17. It can be observed that the tag voltage is approximately $1 \mathrm{~V}$ and the resonance frequency varied with the mutual impedance. Thus a variation in the resonance frequency will cause a change in the inductor series resistance. Therefore, in case of frequency deviation at the generator, the resistance losses can change and may alter the measurement data. As a consequence, variable capacitors $C_{1}$ and $C_{2}$ were 
added to adjust the tag voltage level at the same operation frequency. That procedure maintain the inductor characteristics regardless any frequency variation. Table 2 shows a comparison between free space measurement and simulation data.

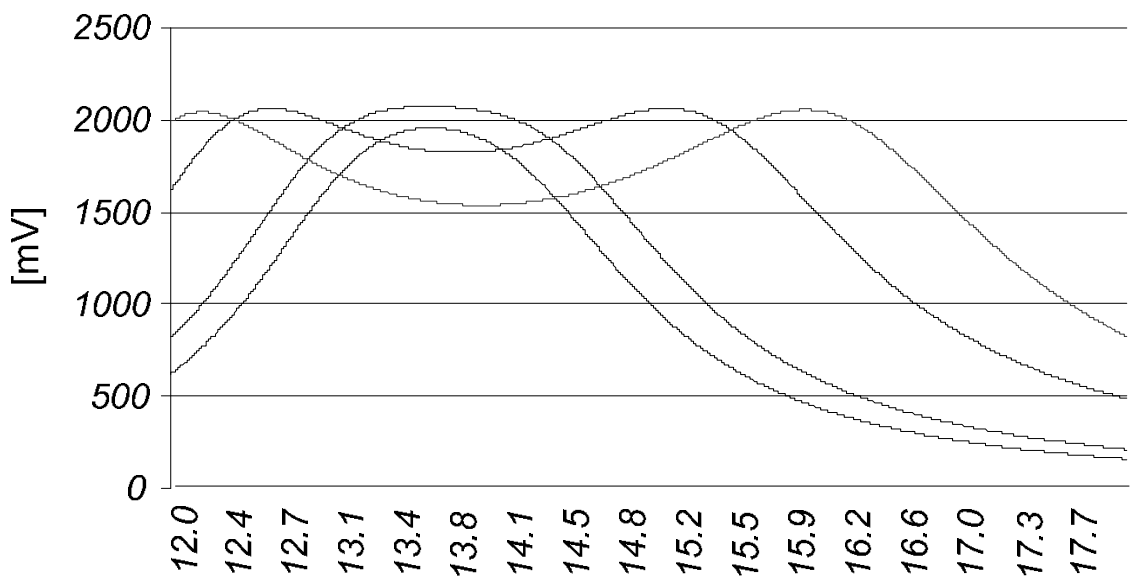

Frequency $[\mathrm{MHz}]$

Figure 17. MATLAB simulation.

\begin{tabular}{|c|c|c|c|}
\hline Distance & Measured & Simulated & $M$ \\
\hline $25 \mathrm{~mm}$ & $769 \mathrm{mV}$ & $1001 \mathrm{mV}$ & $0.287 \mathrm{uH}$ \\
\hline $20 \mathrm{~mm}$ & $943 \mathrm{mV}$ & $1061 \mathrm{mV}$ & $0.370 \mathrm{uH}$ \\
\hline $15 \mathrm{~mm}$ & $1038 \mathrm{mV}$ & $1063 \mathrm{mV}$ & $0.532 \mathrm{uH}$ \\
\hline $10 \mathrm{~mm}$ & $1026 \mathrm{mV}$ & $1055 \mathrm{mV}$ & $0.692 \mathrm{uH}$ \\
\hline $5 \mathrm{~mm}$ & $1007 \mathrm{mV}$ & $1052 \mathrm{mV}$ & $0.917 \mathrm{uH}$ \\
\hline
\end{tabular}

Table 2. Comparison between free space measurement and simulation data.

As it can be observed, there is an error between the measurement and the simulation data, as it is shown in Table 3. Note that the error is smaller than $4.3 \%$ for distances smaller than $15 \mathrm{~mm}$. The error becomes larger for distance over $15 \mathrm{~mm}$, since there is magnetic field scattering due to coil imperfections. The connection cables between generator and reader may cause a magnetic field distribution, thus increasing the error.

\begin{tabular}{|c|c|}
\hline Distance & Error \\
\hline $5 \mathrm{~mm}$ & $-4.3 \%$ \\
\hline $10 \mathrm{~mm}$ & $-2.75 \%$ \\
\hline $15 \mathrm{~mm}$ & $-2.34 \%$ \\
\hline $20 \mathrm{~mm}$ & $-11.12 \%$ \\
\hline $25 \mathrm{~mm}$ & $-23.17 \%$ \\
\hline
\end{tabular}

Table 3. Error between measurement and simulation data for free space. 


\section{Comparison between free space and animal tissue}

Once the tag voltage for free space is known, it can be compared to implanted conditions, where there is tissue between reader and tag. Figure 18 shows the set up used for the measurements, and Table 4 provides the tag voltage comparison between free space and pork tissue conditions. Since pork tissue presents electromagnetic properties similar to human tissue, it will be used in the measurements.

\begin{tabular}{|c|c|c|}
\hline Distance & Free Space & Pork Tissue \\
\hline $10 \mathrm{~mm}$ & $1932 \mathrm{mV}$ & $1869 \mathrm{mV}$ \\
\hline $15 \mathrm{~mm}$ & $1869 \mathrm{mV}$ & $1787 \mathrm{mV}$ \\
\hline $20 \mathrm{~mm}$ & $1671 \mathrm{mV}$ & $1549 \mathrm{mV}$ \\
\hline $25 \mathrm{~mm}$ & $1247 \mathrm{mV}$ & $1202 \mathrm{mV}$ \\
\hline
\end{tabular}

Table 4. Comparison between free space and pork tissue data.

The generator voltage was raised to approximately $1.94 \mathrm{~V}$ in order to assure that low voltage measurements are not spoiled by noise. The pork tissue thickness is approximately $10 \mathrm{~mm}$. As it can be directly observed form Table 4, the free space data is different form the pork tissue data. The small difference suggests that at $13.56 \mathrm{MHz}$ the pork tissue causes s variation in the magnetic permeability as compared to free space. The error between free space and pork tissue data is presented in Table 5 . The $5 \mathrm{~mm}$ measurement was not conducted due to the pork tissue thickness The first measurement, $10 \mathrm{~mm}$, was conducted with the reader in direct contact with the pork tissue.

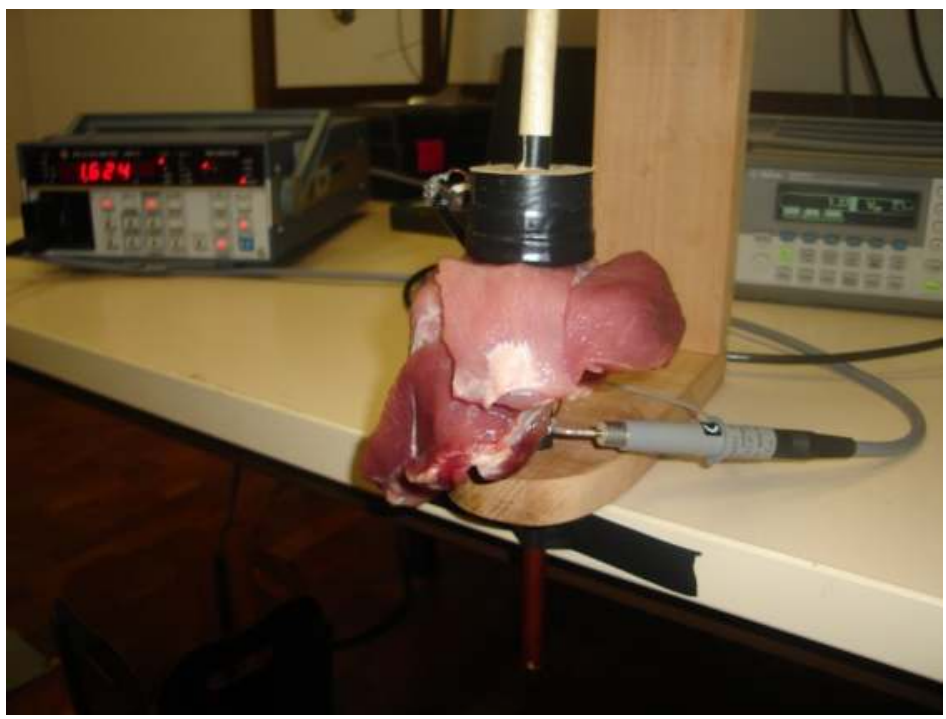

Figure 18. Test set up using pork tissue. 


\begin{tabular}{|c|c|}
\hline Distance & Error \\
\hline $10 \mathrm{~mm}$ & $-3.26 \%$ \\
\hline $15 \mathrm{~mm}$ & $-4.39 \%$ \\
\hline $20 \mathrm{~mm}$ & $-7.30 \%$ \\
\hline $25 \mathrm{~mm}$ & $-3.61 \%$ \\
\hline
\end{tabular}

Table 5. Error between free space and pork tissue data.

\section{Conclusions}

The classic circuit theory can be used to obtain the tag voltage from the mutual inductance between reader and tag in a RFID system. Therefore, based on the estimate of the mutual inductance between reader and tag, it is possible to estimate voltage at the tag. Nevertheless the main distress is the variation on the resonance frequency due to the mutual inductance, and ultimately, the coupling factor.

Two approximations must be conducted to estimate the tag voltage. The first one is due to the magnetic scattering between reader and tag inductors that becomes more relevant as the distance between them increases. The second approximation is due to the tissue that alters the magnetic permeability and consequently reduces the tag voltage. Thus, by considering the simulation errors and the errors due to the tissue, it is possible to estimate the voltage at the tag. Table 6 presents the compiled errors.

\begin{tabular}{|c|c|}
\hline Distance & Error \\
\hline $10 \mathrm{~mm}$ & $-5.92 \%$ \\
\hline $15 \mathrm{~mm}$ & $-6.66 \%$ \\
\hline $20 \mathrm{~mm}$ & $-17.60 \%$ \\
\hline $25 \mathrm{~mm}$ & $-26.90 \%$ \\
\hline
\end{tabular}

Table 6. Total errors between simulated and measured data.

Therefore, by taking into account both approximations it is possible to estimate the voltage drop at the tag and consequently, the power reduction. Once the power reduction is known, it can be taken into account during the tag circuit design. Consequently, the use of classic theory of magnetically coupled circuits is a simple way to estimate the voltage in implanted devices. Nevertheless additional studies regarding the resonance frequency variation is still required.

\section{Author details}

Paulo Cesar Crepaldi, Tales Cleber Pimenta and Robson Luiz Moreno Universidade Federal de Itajuba, Brasil

Rômulo Mota Volpato Inatel Santa Rita do Sapucai, Brasil 


\section{References}

[1] M. M. Ahmadi, G. A. Julien, “A Wireless Implantable Microsytem for Continuous Blood Glucose Moritoring", IEE Transations on Biomedical and Systems, Vol 3, No. 3 June 2009 Pag 169-180.

[2] F. Ramos, M. Santana, R. M. Volpato, R. L. Moreno and T. C. Pimenta, “Front -End of an Implantable Medical Device", Wireless Systems International Meeting, May 26-28, 2010 Campina Grande , Brazil.

[3] TESLA Q-METRO Model BM 409 Service manual.

[4] F. E. Terman, J. M. Pettit, Electronic Measurements, MacGraw-Hill 1952.

[5] K. Finkenzeller, RFID Handbook - Fundamental and Application in Contactless Smart Cards and Identifications, 2010, John Wiley \& Sons.

[6] Mehdi Kiani, Maysam Ghovanloo, “An RFID-Based Closed-Loop Wireless Power Transmission System for Biomedical Applications", IEEE Transactions on Circuits and Systems -II: Express Briefs, Vol. 57, No. 4, April 2010. 